\title{
Sensitivity to anomalous quartic gauge couplings in photon-photon interactions at the LHC
}

Contribution to the CERN workshop on "High energy photon collisions at the LHC", 22-25 $5^{\text {th }}$ April 2008

\author{
T. Pierzchała ${ }^{a *}$ and K. Piotrzkowski ${ }^{\mathrm{a}}$ \\ ${ }^{a}$ Université catholique de Louvain, Center for Particle Physics and Phenomenology (CP3) \\ Chemin du Cyclotron 2, 1348 Louvain-la-Neuve, Belgium
}

\begin{abstract}
The exclusive two-photon production at the LHC of pairs of $\mathrm{W}$ and $\mathrm{Z}$ bosons provides a novel and unique testground for the electroweak gauge boson sector. In particular it offers, thanks to high $\gamma \gamma$ center-of-mass energies, large and direct sensitivity to the anomalous quartic gauge couplings otherwise very difficult to investigate at the LHC. An initial analysis has been performed assuming leptonic decays and generic acceptance cuts. Simulation of a simple counting experiment has shown for the integrated luminosity of $10 \mathrm{fb}^{-1}$ at least four thousand times larger sensitivity to the genuine quartic couplings, $a_{0}^{W}, a_{0}^{Z}, a_{C}^{W}$ and $a_{C}^{Z}$, than those obtained at LEP. The impact of the unitarity constraints on the estimated limits has been studied using the dipole form-factors. Finally, differential distributions of the decay leptons have been provided to illustrate the potential for further improvements of the sensitivities.
\end{abstract}

\section{LHC as a photon collider}

The $\gamma \gamma$ exclusive production of pairs of charged particles offers interesting potential for signals of new physics at the LHC. In a recent paper [1, the initial comprehensive studies of high energy photon interactions at the $\mathrm{LHC}$ were reported. In the present contribution, the selected results on the gauge boson pairs discussed in Ref. [1] are introduced and supplemented by new results.

The exclusive two-photon production, $p p \rightarrow$ $p X p$, provides clean experimental conditions, thanks to absence of the proton remnants. Well defined final states can be then selected, and precisely reconstructed. Moreover, detection of the two final state protons, scattered at almost zerodegree angle, in the dedicated very forward detectors (VFDs), provides another striking signature, effective also at high luminosity and with large event pile-up [23]. In addition, the photon energies can be then measured and used for the event kinematics reconstruction. Finally, virtualities of the exchanged photons are on average very small, and are limited from above due to the

\footnotetext{
*Email: Tomasz.Pierzchala@uclouvain.be
}

proton electromagnetic form-factors, allowing for treating the LHC protons as sources of quasi-real photons.

The cross sections of two-photon pair production are in general determined by the mass, spin and charge of the produced particles, so the rate of produced particles at the LHC can be well predicted using the Equivalent Photon Approximation for the equivalent photon fluxes [4]. For proton-proton collisions at $\sqrt{s}=14 \mathrm{TeV}$ the EPA predicts the photon-photon luminosity spectrum as shown in Fig. 1, where $d L_{\gamma \gamma} / d W_{\gamma \gamma}$ is defined by the relation between the proton-proton and $\gamma \gamma$ cross-sections assuming a minimal center-of-mass energy $W_{0}$ :

$$
\sigma_{p p}=\int_{W_{0}}^{\sqrt{s}} \sigma_{\gamma \gamma} \frac{d L_{\gamma \gamma}}{d W_{\gamma \gamma}} d W_{\gamma \gamma} .
$$

A set of VFDs at $220 \mathrm{~m}$ or $420 \mathrm{~m}$ from the LHC interaction points will be capable of tagging photon interactions within the wide photon energy range of $20 \mathrm{GeV}<E_{\gamma}<900 \mathrm{GeV}$ [5]6]. In Fig. 1 also the $\gamma \gamma$ luminosity spectrum is shown assuming double tagging (i.e. requesting both forward protons to be detected). One should note 
quoted.

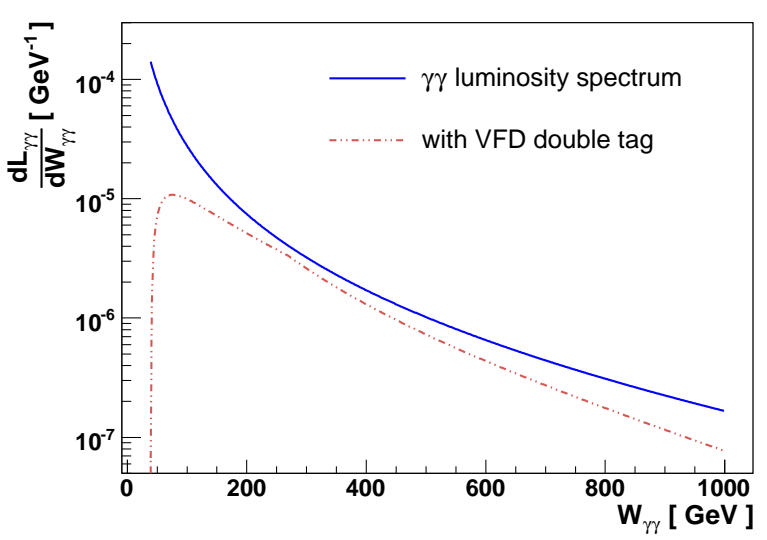

Figure 1. Elastic luminosity spectrum of photonphoton collisions at the LHC assuming the maximal photon virtuality $Q_{\max }^{2}=2 \mathrm{GeV}^{2}$ (solid line). The luminosity spectrum assuming the photon tagging range $20 \mathrm{GeV}<E_{\gamma}<900 \mathrm{GeV}$ is also shown (dashed line).

that apart from such elastic two-photon processes where both protons have survived the interaction, the inelastic production can also be considered, when at least one of the two protons dissociates into a low mass state. The corresponding twophoton luminosity increases then by about a factor of three [2]. By integrating the luminosity spectrum above some minimal center-of-mass energy $W_{0}$, one can introduce the relative photonphoton luminosity $L_{\gamma \gamma}$, shown in Fig. 2. Effectively, $L_{\gamma \gamma}$ gives a fraction of the proton-proton luminosity which is available for $\gamma \gamma$ collisions, and is especially useful if a given photon-photon cross-section is approximately constant as a function of $W_{\gamma \gamma}$. For example, the relative photonphoton luminosity at the $\mathrm{LHC}$ is equal to $1 \%$ for $W_{0}=23 \mathrm{GeV}$ (i.e. for $W_{\gamma \gamma}>23 \mathrm{GeV}$ ), and $0.1 \%$ for $W_{0}=225 \mathrm{GeV}$. Given the very large LHC luminosity, this leads to significant event rates of high-energy processes with relatively small photon-photon cross sections. In the following only the proton cross-sections are



Figure 2. Relative elastic $\gamma \gamma$ luminosity for photon collisions at the center-of-mass energy above $W_{0}$, obtained using the EPA for proton collisions at $\sqrt{s}=14 \mathrm{TeV}$, and assuming the maximal photon virtuality $Q_{\max }^{2}=2 \mathrm{GeV}^{2}$.

One needs however to consider corrections beyond the EPA due to a possibility of strong interactions between protons, or the so-called rescattering effects. The resulting suppression of the cross sections weakly depends on the invariant mass of the exclusively produced state $X$, and for the processes discussed in the following, as $p p \rightarrow p W W p$, it is estimated to be about $15 \%$ [7. This correction is ignored in the present analysis. In addition, one should stress that potentially dangerous background due to the exclusive diffractive production is heavily suppressed for non strongly-interacting particles and can be safely neglected. For example, the gluon mediated exclusive production of $\mathrm{W}$ boson pairs, is about 100 times smaller than the two-photon production at the LHC [8].

Anomalous quartic couplings of the electroweak gauge bosons could directly reveal the exchange of new heavy bosons 910, and the 
two-photon exclusive production of $\mathrm{WW}$ and $\mathrm{ZZ}$ pairs is particularly well suited for studies of the quartic couplings $\gamma \gamma W W$ and $\gamma \gamma Z Z$. In the Standard Model (SM), the cross section of the exclusive two-photon production $p p \rightarrow p W W p$ is large about $108 \mathrm{fb}^{-1}[1$. This means that unique, high statistics tests of the gauge boson sector in the SM can be performed at the LHC. In contrast, the cross section of the exclusive two-photon production $p p \rightarrow p Z Z p$ is very small in the $\mathrm{SM}$, since this proccess is not allowed at the tree level. It means that an observation of even a few events of this type could signal the new physics.

\section{Anomalous quartic gauge couplings}

The anomalous quartic gauge couplings (AQGCs) can be introduced in various ways, usually by building an effective lagrangian which models a low energy behavior of a wide class of possible extensions of the SM. For example, one can introduce new terms in such a lagrangian, which are allowed by the local $\mathrm{U}(1)_{Y} \times \mathrm{SU}(2)_{L}$ gauge invariance, and then consider two scenarios, with [11 and without [12 the Higgs boson. In the present analysis, the phenomenological lagrangians are used, which allow for genuine anomalous quartic vector boson couplings, without need for associated trilinear gauge couplings. First, the simplest lagrangian term of power six in energy for two photon interaction with weak bosons has to conserve local $\mathrm{U}(1)_{e m}$ and custodial $\mathrm{SU}(2)_{c}$. Then, by imposing conservation of discrete $\mathrm{C}$ and $\mathrm{P}$ symmetries one finally obtains two new terms [913]:

$L_{6}^{0}=-\frac{e^{2}}{16} \frac{a_{0}}{\Lambda^{2}} F_{\mu \nu} F^{\mu \nu} \vec{W}^{\alpha} \cdot \vec{W}_{\alpha}$

$L_{6}^{C}=-\frac{e^{2}}{16} \frac{a_{C}}{\Lambda^{2}} F_{\mu \alpha} F^{\mu \beta} \vec{W}^{\alpha} \cdot \vec{W}_{\beta}$,

where $e$ is the electron charge and $\Lambda$ is the energy scale of the new physics. When the global $\mathrm{SU}(2)_{c}$ symmetry is not imposed then one can distinguish the neutral and charged couplings of the $\mathrm{W}$ and $\mathrm{Z}$ bosons. Since this more general model has been assumed for studies at LEP [14, to allow for a direct comparison of sensitivities, this is also assumed in the following:

$$
\begin{aligned}
\mathcal{L}_{6}^{0}= & -\frac{e^{2}}{8} \frac{\mathbf{a}_{0}^{\mathrm{W}}}{\mathbf{\Lambda}^{\mathbf{2}}} F_{\mu \nu} F^{\mu \nu} W^{+\alpha} W_{\alpha}^{-} \\
& -\frac{e^{2}}{16 \cos ^{2} \theta_{W}} \frac{\mathbf{a}_{\mathbf{0}}^{\mathrm{Z}}}{\mathbf{\Lambda}^{\mathbf{2}}} F_{\mu \nu} F^{\mu \nu} Z^{\alpha} Z_{\alpha}, \\
\mathcal{L}_{6}^{\mathrm{c}}= & -\frac{e^{2}}{16} \frac{\mathbf{a}_{\mathrm{c}}^{\mathrm{W}}}{\mathbf{\Lambda}^{\mathbf{2}}} F_{\mu \alpha} F^{\mu \beta}\left(W^{+\alpha} W_{\beta}^{-}+W^{-\alpha} W_{\beta}^{+}\right) \\
& -\frac{e^{2}}{16 \cos ^{2} \theta_{W}} \frac{\mathbf{a}_{\mathrm{c}}^{\mathrm{Z}}}{\mathbf{\Lambda}^{\mathbf{2}}} F_{\mu \alpha} F^{\mu \beta} Z^{\alpha} Z_{\beta} .
\end{aligned}
$$

Using this formalism, one obtains a general relation for the $W W$ and $Z Z$ cross sections (which is also valid after applying the acceptance cuts) as a function of the anomalous parameters:

$\sigma=\sigma_{S M}+\sigma_{0} a_{0}+\sigma_{00} a_{0}^{2}+\sigma_{c} a_{c}+\sigma_{c c} a_{c}^{2}+\sigma_{0 c} a_{0} a_{c}$

which corresponds, for a fixed cross section $\sigma$, to an ellipse on the $a_{0}, a_{c}$ plane.

\section{Estimation of sensitivity}

Simulation of the exclusive two-photon pair production at the LHC was performed using the modified MadGraph/MadEvent [16] and CalcHep [17. packages. The generated events were then passed to the modified Pythia generator [18] to allow for decays and hadronisation. The sensitivity to the anomalous quartic vector boson couplings at the LHC has been investigated using the signature of two opposite charge leptons $(e$ or $\mu)$ within the generic lepton acceptance window $-|\eta|<2.5$ and $p_{T}>10 \mathrm{GeV}$. In the $W W$ case it corresponds to the subprocesses $\gamma \gamma \rightarrow W^{+} W^{-} \rightarrow l^{+} l^{-} \nu \bar{\nu}$ while in the $Z Z$ case to the subprocesses $\gamma \gamma \rightarrow Z Z \rightarrow l^{+} l^{-} j j$. It is assumed that both processes are background free. Under this condition, the upper limits of number of events $\lambda^{u p}$ at the $95 \%$ confidence level (CL) were calculated assuming the number of observed events equal to the $S M$ prediction $\mathrm{N}_{\text {obs }}=\sigma_{\text {acc }}^{\mathrm{SM}} \mathrm{L}$ for a given integrated luminosity $\mathrm{L}$.

$\sum_{\mathrm{k}=0}^{\mathrm{N}_{\mathrm{obs}}} \mathrm{P}_{\text {Poisson }}\left(\lambda^{\mathrm{up}}=\sigma^{\mathrm{up}} \mathrm{L} ; \mathrm{k}\right)=1-\mathrm{CL}$

This is a simplified approach and for more precise analysis one should use formula from [15], however the difference in the obtained limits is small, less than $20 \%$. 
The expected $95 \%$ CL limits $\lambda^{\text {up }}$ have been then used to calculate the upper limits on the observed cross section $\sigma^{\text {up }}$ at the $95 \%$ CL for the integrated luminosity $\mathrm{L}=1 \mathrm{fb}^{-1}$ and $\mathrm{L}=10 \mathrm{fb}^{-1}$, shown in the Table 1.

Table 1

Expected 95\% CL upper limits for the cross sections after acceptance cuts for chosen subprocesses $\gamma \gamma \rightarrow W^{+} W^{-} \rightarrow l^{+} l^{-} \nu \bar{\nu}$ and $\gamma \gamma \rightarrow Z Z \rightarrow$ $l^{+} l^{-} j j$. The assumed numbers of the observed $W W$ events correspond to the $S M$ cross section calculated using $\mathrm{MG} / \mathrm{ME}$ and the acceptance cuts.

\begin{tabular}{ccc}
\hline$\sigma^{\text {up }}[\mathrm{fb}]$ & $\gamma \gamma \rightarrow W^{+} W^{-}$ & $\gamma \gamma \rightarrow Z Z$ \\
& & $N_{\text {obs }}=0$ \\
& $\sigma_{\mathrm{acc}}^{\mathrm{SM}}=4.081 \mathrm{fb}$ & $\lambda^{\text {up }}=2.996$ \\
\hline $\mathrm{L}=1 \mathrm{fb}^{-1}$ & 9.2 & 3.0 \\
$\mathrm{~L}=10 \mathrm{fb}^{-1}$ & 5.3 & 0.30 \\
\hline
\end{tabular}

The calculated cross section upper limits can be directly converted to the limits of the anomalous quartic couplings as presented at the Fig. 3 where the 95\% CL contours are shown. In Fig. 4. one parameter limits (with the other anomalous coupling set to zero) are shown, and the obtained limits are quoted in the Table 2.

Table 2

Expected one-parameter limits for anomalous quartic vector boson couplings at 95\% CL for two values of the integrated luminosity.

\begin{tabular}{ccc}
\hline Coupling & \multicolumn{2}{c}{ Limits $\left[10^{-6} \mathrm{GeV}^{-2}\right]$} \\
$\mathrm{L}=1 \mathrm{fb}^{-1}$ & $\mathrm{~L}=10 \mathrm{fb}^{-1}$ \\
\hline$\left|\mathrm{a}_{0}^{\mathrm{Z}} / \Lambda^{2}\right|$ & 0.49 & 0.16 \\
$\left|\mathrm{a}_{\mathrm{C}}^{\mathrm{Z}} / \Lambda^{2}\right|$ & 1.84 & 0.58 \\
$\left|\mathrm{a}_{0}^{\mathrm{W}} / \Lambda^{2}\right|$ & 0.54 & 0.27 \\
$\left|\mathrm{a}_{\mathrm{C}}^{\mathrm{W}} / \Lambda^{2}\right|$ & 2.02 & 0.99 \\
\hline
\end{tabular}

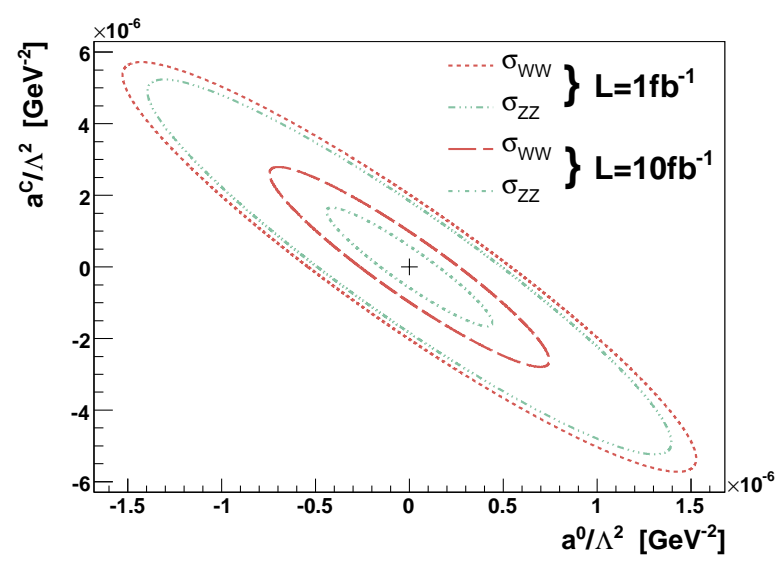

Figure 3. Profiles of the 95\% CL upper limits of the cross section after the acceptance cuts for $p p\left(\gamma \gamma \rightarrow W^{+} W^{-} \rightarrow l^{+} l^{-} \nu \bar{\nu}\right) p p$ and $p p(\gamma \gamma \rightarrow$ $\left.Z Z \rightarrow l^{+} l^{-} j j\right) p p$ as a function of relevant anomalous couplings $\mathrm{a}_{0}^{\mathrm{W}} / \Lambda^{2}, \mathrm{a}_{\mathrm{c}}^{\mathrm{W}} / \Lambda^{2}, \mathrm{a}_{0}^{\mathrm{Z}} / \Lambda^{2}$ and $\mathrm{a}_{\mathrm{c}}^{\mathrm{Z}} / \Lambda^{2}$. The contours are shown assuming two values of the integrated $p p$ luminosity.

The obtained limits are about 40000 times better than the best limits established at LEP2 14 clearly showing large and unique potential of such studies at the LHC.

\section{Impact of unitarity bound condition}

The lagrangian terms in Eq.(2), do not preserve the $\mathrm{SU}(2)_{L}$ local symmetry. In consequence, for the center-of-mass energies $W_{\gamma \gamma} \gg 2 M_{W}$ the scattering amplitude will grow and eventually will violate the unitarity condition. It is therefore necessary to investigate the impact of the unitarity constraint on the derived limits. For simplicity, the unitarity violation is checked only for the process $\gamma \gamma \rightarrow W^{+} W^{-}$. The $\gamma \gamma \rightarrow \gamma \gamma$ and $\gamma \gamma \rightarrow Z Z$ (i.e. the anomalous coupling $\gamma \gamma Z Z$ is set to zero) processes are neglected, as well as the two-photon exclusive production of fermion pairs as it drops fast with $W_{\gamma \gamma}$ [1]. For spin one particles one obtains the partial wave amplitudes $a_{J}$ using the 


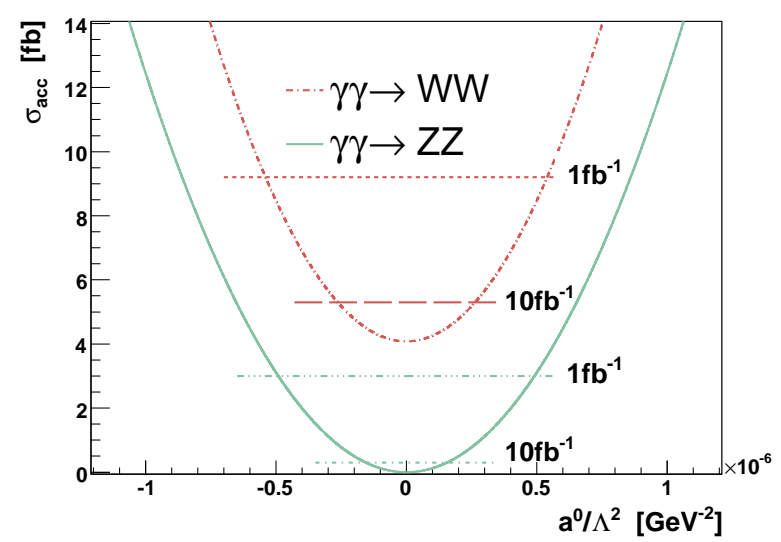

Figure 4. Cross sections of $p p\left(\gamma \gamma \rightarrow W^{+} W^{-} \rightarrow\right.$ $\left.l^{+} l^{-} \nu \bar{\nu}\right) p p$ and $p p\left(\gamma \gamma \rightarrow Z Z \rightarrow l^{+} l^{-} j j\right) p p$ after the acceptance cuts are shown as a function of the genuine anomalous quartic vector boson couplings $\mathrm{a}_{0}^{\mathrm{W}} / \Lambda^{2}$ and $\mathrm{a}_{0}^{\mathrm{Z}} / \Lambda^{2}$ (for $\mathrm{a}_{\mathrm{C}}^{\mathrm{W}} / \Lambda^{2}=$ $\left.\mathrm{a}_{\mathrm{C}}^{\mathrm{Z}} / \Lambda^{2}=0\right)$, together with the upper cross section limits at $\mathrm{CL}=95 \%$ (horizontal lines).

Legendre polynomial $P_{J}$ :

$a_{J}(\sqrt{s})=\frac{1}{32 \pi} \int_{-1}^{1} d u \mathcal{M}\left(\sqrt{s}, u, a_{0}^{W}, a_{C}^{W}\right) P_{J}(u)$,

where $u$ is the cosine of the $\mathrm{W}$ boson polar angle in the $\gamma \gamma$ center-of-mass system, and $\mathcal{M}$ is the amplitude of the process $\gamma \gamma \rightarrow W^{+} W^{-}$. Then, the unitarity bound condition has a form:

$\beta \sum_{p o l}\left|a_{J}\left(\sqrt{s}, a_{0}^{W}, a_{C}^{W}\right)\right|^{2} \leq(1 / 2)^{2}$

where $\beta=\sqrt{1-4 m_{W}^{2} / s}$ is the Lorentz velocity of a $\mathrm{W}$ boson in the cms frame and pol stands for polarization states in $\gamma \gamma \rightarrow W^{+} W^{-}$.

Unitarity bounds of Eq. 6 for $\mathrm{J}=0$ as a function of $W_{\gamma \gamma}$ are shown for both neutral, in Fig. 5 , and charged, in Fig. 6. AQGCs together with the obtained limits of anomalous couplings for the integrated luminosity $L=10 \mathrm{fb}^{-1}$. It shows that for these limits the unitarity will be violated when $W_{\gamma \gamma}$ is above $2 \mathrm{TeV}$, also if one allows for a contribution of some other channels, by requesting the

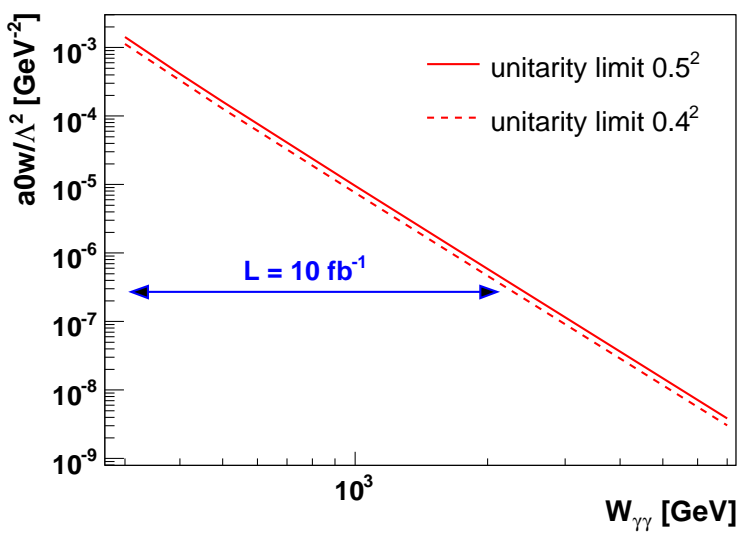

Figure 5. Unitarity limit for the $\mathrm{W}$ neutral anomalous coupling $a_{0}^{W} / \Lambda^{2}$ calculated according to Eq. [6 for $\mathrm{J}=0$ (full line), and assuming that the left hand side of Eq. [6 is smaller than $(0.4)^{2}$ (dashed line). The arrow indicates the obtained limit at 95\% CL from Tab. 2 for $10 \mathrm{fb}^{-1}$.

left hand side of Eq. 6 to be smaller than $(0.4)^{2}$ instead of $(0.5)^{2}$. Other $W W$ states with different total angular momentum do not violate the unitarity earlier, and $a_{1}$ is close to 0 when $a_{2}$ is much smaller than $a_{0}$.

The unitarity violation occurs due to the increasing contribution of longitudinally polarized $\mathrm{W}$ bosons, which in the SM is fully compensated thanks to the local $\mathrm{SU}(2)_{L}$ symmetry and the trilinear gauge couplings. As a consequence, one has to verify if the limits quoted in Tab. 2 are not driven by $\gamma \gamma$ interactions at $W_{\gamma \gamma}$ above $2 \mathrm{TeV}$. As one can see in Fig. [7 this is actually the case, since the anomalous cross-section is large for the energies well above $2 \mathrm{TeV}$. This can be avoided in two ways. The double tagging using very forward detectors would limit the maximal $\gamma \gamma$ center-ofmass energy to $1.8 \mathrm{TeV}[\underline{6}$. One should note that the tagging efficiency in this energy range is high, about $80 \%$ [19. In other case, one can introduce dipole form-factors for each anomalous coupling, as in Eq.7. The form-factors suppress the anoma- 


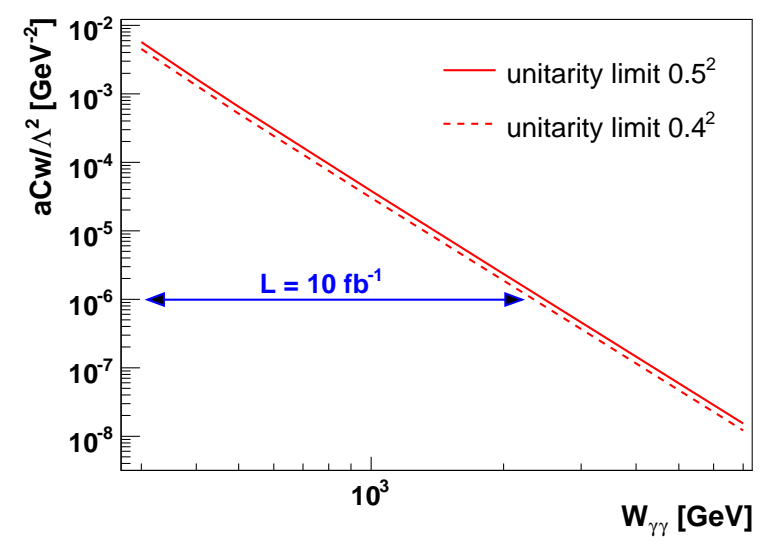

Figure 6. Analogous plot to Fig. 5 but for the $\mathrm{W}$ charged anomalous coupling $a_{C}^{W} / \Lambda^{2}$. Also in this case, unitarity will be violated for $W_{\gamma \gamma}$ above $2 \mathrm{TeV}$.

lous amplitudes when the energies are close to the new physics energy scale $\Lambda$ :

$\mathbf{a} \rightarrow \frac{\mathbf{a}}{\left(1+W_{\gamma \gamma}^{2} / \Lambda^{2}\right)^{X}}$,

where the power $X$ is chosen so to preserve the unitarity - in the present analysis $X=2$.

\section{Expected limits for anomalous quartic gauge couplings}

The sensitivity analysis has been repeated for the process $\gamma \gamma \rightarrow W^{+} W^{-}$using the above formfactors. In Fig. 8 and in Fig. 9 the new sensitivity limits for the integrated luminosity $L=10 \mathrm{fb}^{-1}$ are shown.

The limits were calculated for 5 different $\Lambda$ values and linearly interpolated. Moreover, the unitarity limit (solid line in Figs. 819) is calculated at $W_{\gamma \gamma}=\Lambda$, after correcting for the form-factor suppression. This choice can be explained in the following way. If one obtains a limit of an anomalous coupling for $W_{\gamma \gamma}=\Lambda$ than for the energies below $\Lambda$ the unitarity is automatically preserved. For higher energies, bigger than $\Lambda$ the form-factor

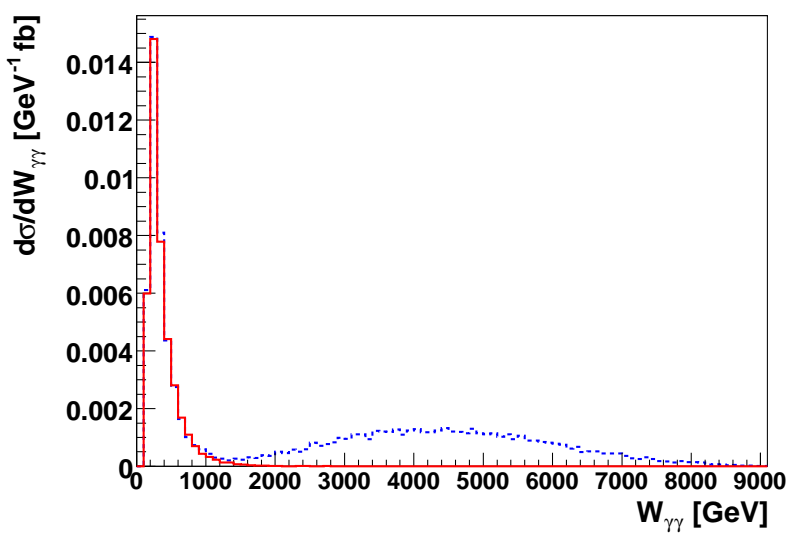

Figure 7. Differential cross section $d \sigma / d W_{\gamma \gamma}$ for $\gamma \gamma \rightarrow W^{+} W^{-} \rightarrow l^{+} l^{-}$after acceptance cuts, for the SM (solid line) and for the AQGC case (dashed line), assuming $a_{0}^{W} / \Lambda^{2}=0.54$. $10^{-6} \mathrm{GeV}^{-2}$ and $a_{C}^{W} / \Lambda^{2}=0$.

suppression should be strong enough to keep the unitarity unbroken.

By repeating the sensitivity analysis from Sec. 3, the results shown in Fig. 8 and in Fig. 9 are obtained, and the following 95\% CL limits for the anomalous quartic couplings can be derived for $\gamma \gamma \rightarrow W^{+} W^{-}$fully leptonic events, for the integrated luminosity $L=10 \mathrm{fb}^{-1}$ :

$a_{0}^{W} / \Lambda^{2}<2.5 \cdot 10^{-6} \mathrm{GeV}^{-2}$

$a_{C}^{W} / \Lambda^{2}<9 \cdot 10^{-6} \mathrm{GeV}^{-2}$,

which are about 4000 times stronger than achieved at LEP [14, and are 10 times weaker than results from Tab. 2, obtained ignoring the unitarity constraints.

\section{Summary and perspectives}

The initial analysis of sensitivity to genuine AQGCs in photon-photon collisions at the LHC has been presented. A simple method of event counting has provided very promising results, showing improvements of the LEP AQGC limits 


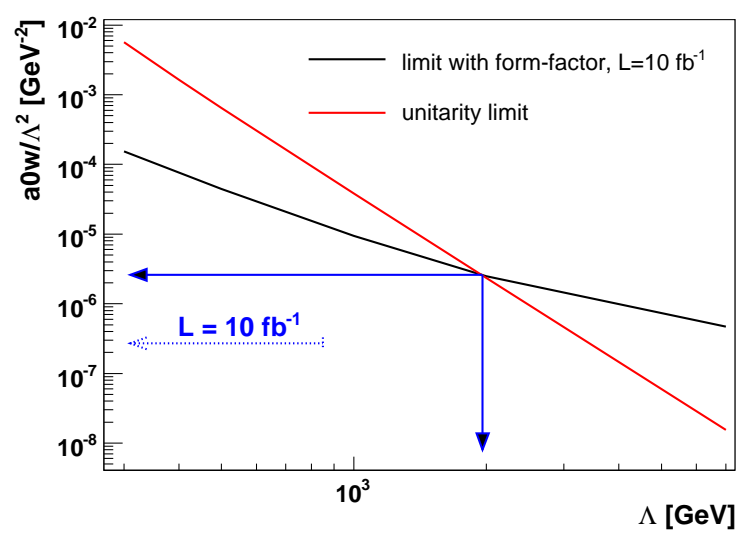

Figure 8. The 95\% CL limits of the W neutral anomalous quartic coupling (dashed line) calculated for the integrated luminosity $L=10 \mathrm{fb}^{-1}$ with the dipole form-factor. The unitarity curve (solid line) is calculated for $W_{\gamma \gamma}=\Lambda$, and due to inclusion of the from-factor is four times higher than in Fig. 5 . The solid arrows indicate the strongest limit without violating the unitarity, and the corresponding energy scale $\Lambda$ of the new physics. Dashed arrow recalls the limit obtained without the form-factors.

by a factor of about 4000 , for the integrated luminosity $10 \mathrm{fb}^{-1}$. The effects of the form-factors, introduced to preserve unitarity constraints, are taken into account. In addition, the analysis demonstrates the sensitivity to the new physics energy scale up to $2 \mathrm{TeV}$.

There are still several possible ways to improve the sensitivity. First, one can include also semileptonic channels in $\gamma \gamma \rightarrow W W$, which will increase statistics about 6 times.

In addition, one can increase sensitivity by studing the differential distributions, like the lepton pseudo-rapidity (see Fig. 10), or the lepton acoplanarity $\delta \phi=\pi-\operatorname{Min}(2 \pi-\Delta \phi, \Delta \phi$ ) (see Fig. 11) distributions. Finally, assuming the custodial SU $(2)_{c}$ symmetry, one can convert the limits of $a_{0}^{W}$ and $a_{0}^{Z}$ into the single $a_{0}$ limit, and

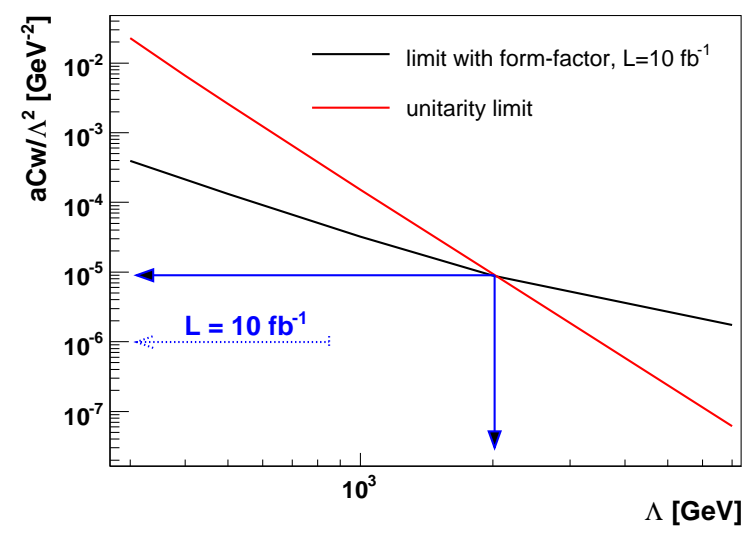

Figure 9. The sensitivity plot for the $\mathrm{W}$ charged anomalous quartic coupling. The plot is analogous to Fig. 8 ,

similarly the $a_{C}^{W}$ and $a_{C}^{Z}$ limits into the single $a_{C}$ limit (see. Eq. 1). This will be the subject of a future publication.

\section{REFERENCES}

1. J. de Favereau et al., High energy photon interactions at the LHC, CP3-08-04, July 2008, to be submitted to EPJC.

2. K. Piotrzkowski, Phys. Rev. D 63 (2001) 071502.

3. M. Albrow et al., The FP420 R\&D Project: Higgs and New Physics with forward protons at the LHC FP420, arXiv:0806.0302 [hep-ex].

4. V. M. Budnev et al., Phys. Rept. 15 (1974) 181.

5. J. de Favereau de Jeneret, X. Rouby and K. Piotrzkowski, JINST 2: P09005 (2007), arXiv:0707.1198v1 [physics.acc-ph] (CP3-0713).

6. X. Rouby, Tagging photon interactions at the $L H C$, these proceedings, arXiv:0806.4941 [hep-ex].

7. V. A. Khoze et al., Eur. Phys. J. C 23 (2002) 311; private communication from V. Khoze.

8. B. E. Cox et al., Eur. Phys. J. C 45 (2006) 


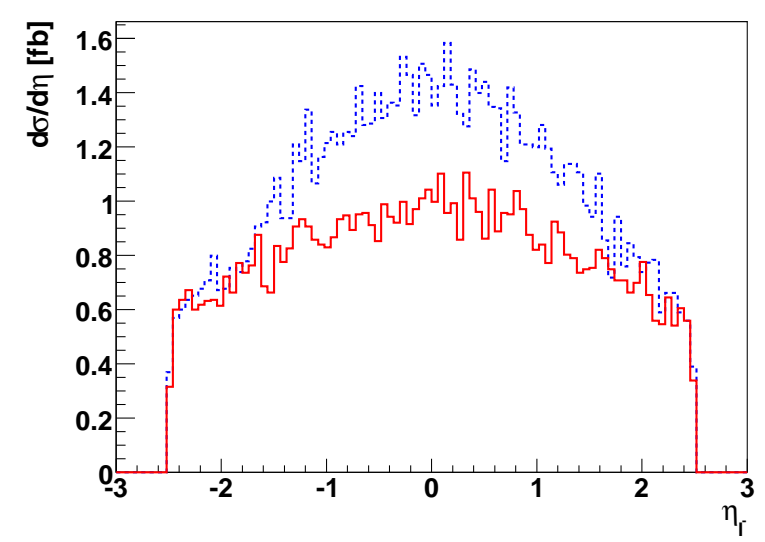

Figure 10. Differential cross sections $d \sigma / d \eta$ for $\gamma \gamma \rightarrow W^{+} W^{-} \rightarrow l^{+} l^{-}$after acceptance cuts for the SM (solid line) and for the anomalous quartic coupling (dashed line), $a_{0}^{W} / \Lambda^{2}=2.5 \cdot 10^{-6} \mathrm{GeV}^{-2}$ and $\Lambda=2 \mathrm{TeV}$.

401.

9. G. Belanger and F. Boudjema, Phys. Lett. B 288 (1992) 201.

10. C. Arzt, M. B. Einhorn and J. Wudka, Nucl. Phys. B 433 (1995) 41.

11. M. Maniatis, A. von Manteuffel and O. Nachtmann, Anomalous couplings in $\gamma \gamma \rightarrow W^{+} W^{-}$at the $L H C$ and $I L C$, these proceedings.

12. O. Eboli, LHC potential to study the quartic electroweak gauge boson couplings, these proceedings.

13. O. J. P. Eboli, M. C. Gonzalez-Garcia, S. M. Lietti and S. F. Novaes, Phys. Rev. D 63 (2001) 075008 arXiv:hep-ph/0009262.

14. G. Abbiendi et al. [OPAL Collaboration], Phys. Rev. D 70 (2004) 032005 arXiv:hep-ex/0402021.

15. G. Zech, Nucl. Instrum. Meth. A 277 (1989) 608.

16. J. Alwall et al., JHEP 0709:028 (2007).

17. A. Pukhov, Nucl. Inst. Meth A 502 (2003) 596.

18. T. Sjöstrand et al., Comput. Phys. Commun.



Figure 11. Differential cross section $d \sigma / d(\delta \phi)$ for $\gamma \gamma \rightarrow W^{+} W^{-} \rightarrow l^{+} l^{-}$after acceptance cuts, for the SM (solid line) and for the anomalous quartic coupling (dashed line), $a_{0}^{W} / \Lambda^{2}=2.5 \cdot 10^{-6} \mathrm{GeV}^{-2}$ with $\Lambda=2 \mathrm{TeV}$.

135 (2001) 238.

19. N. Schul and K. Piotrzkowski, Detection of two-photon exclusive production of supersymmetric pairs at the $\mathrm{LHC}$, these proceedings, arXiv:0806.1097 [hep-ph]. 